\title{
Treatment of Opioid Use Disorder With Buprenorphine- Naloxone at Chainama Hills College Hospital in Lusaka, Zambia: A Case Report
}

\author{
Brian Maila, Anatolii Tsarkov, Petro Petlovanyi, Gaise Kweku, \& Evans Musonda
}

\begin{abstract}
Opioid dependence is a disorder of the central nervous system that results from chronic use of opiates. Opioids cause intense feelings of euphoria, and this is what puts opiate users at risk of recreational use of these substances. There is a desire to increase the dose in order to enhance the effect of opioids, therefore addiction arises, which is a serious medical and social problem. The more opiates come from outside, the less natural opiates are produced, and more opiates are required to create a strong feeling of euphoria, which previously could be achieved with a lower dose. The consequence of a chronic opioid use is the drug tolerance, and abrupt cessation of use causes a serious condition of opioid withdrawal syndrome, indicating the presence of physical dependence. This article describes the experience and the case study of Medication Assisted Therapy (MAT) with buprenorphine and naloxone at Chainama Hills College Hospital in Lusaka, Zambia.
\end{abstract}

Index Terms - opiates, opioid use disorder, naloxone, buprenorphine, medication assisted therapy, MAT, harmful use, addiction medicine

\section{BACKGROUND}

According to the World Drug Report 2019, opioid use disorder remains a major public health concern [1]. In 2017, opioid use disorder accounted for 110,000 (66 per cent) of the 167,000 deaths attributed to drug use disorders [1]. Additional health consequences of untreated opioid use disorders include overdose, end-stage liver disease, psychiatric illnesses, as well as increased risk of Hepatitis C (HCV) and HIV infection [1], [2].

An Opioid Use Disorder is defined by 11 diagnostic criteria, occurring over a 12-month period [3]. Symptoms include taking more of the opioid than intended; failed attempts to stop the opioid; excessive time spent obtaining the opioid; cravings for opioids; failure to fulfill obligations; repetitive interpersonal conflicts; giving up important things for the opioids; using opioids in hazardous situations; and using opioids despite knowing the substance is causing significant emotional or physical consequences, tolerance, and withdrawal. Relative severity (mild, moderate, and severe), is defined by the relative number of symptoms that an individual has [3].

One review article done by Volkow, Frieden, Hyde and

Published on August 6, 2020.

Brian Maila, Chainama Hills College Hospital, University of Zambia (UNZA), Zambia.

(e-mail: brianmaila34@gmail.com)

Anatolii Tsarkov, University of Zambia (UNZA), Zambia.

(corresponding e-mail: anatoliydoc ${ }^{\circledR}$ gmail.com)
Cha (2014) stated that effective treatment and functional recovery are possible through the use of the three Medication assisted therapies (MATs) for treating patients with opioid use disorder: methadone, naltrexone, and buprenorphine. Each of these medications has advantages and disadvantages compared to others [4].

Methadone has been shown to be the most successful longterm treatment option for severe Opioid Use Disorder, as it is associated with reductions in intravenous drug use, crime, HIV risk behaviors and mortality, and is well established in community treatment programs around the world [5]. The principal effects of methadone maintenance are to relieve narcotic craving, suppress the withdrawal syndrome, and block the euphoric effects associated with heroin. However, there is controversy around the idea of using a potent longacting opioid to manage a patient with opioid use disorder. Considering that Methadone is a full opioid agonist, abuse is possible, and with its long half-life, it carries a higher safetyrisk profile than other MATs [6]. Additionally, methadone must be administered at specialty facilities separate from the regular health-care system, presenting access barriers [7]. Furthermore, abrupt or rapid discontinuation is associated with severe and prolonged withdrawal symptoms [8].

Naltrexone can be prescribed and administered by any provider in the USA and does not cause euphoric effects, physical dependence, withdrawal, or respiratory depression and therefore poses no risk of diversion or overdose [7]. However, naltrexone has achieved the least penetration of all medications for opioid use disorder. This can be attributed to three main reasons. Firstly, Naltrexone, the antagonist treatment, is generally thought to be poorly tolerated, which mostly reflects the poor compliance reported among people with opioid use disorder treated with oral naltrexone [7]. Secondly, naltrexone induction requires that patients be fully detoxified to prevent the precipitation of withdrawal (socalled "detox hurdle"), which can require several days of tapering off opioid agonist medications [7]. Lastly, the widespread belief that antagonists are less effective than agonist treatments, despite the lack of comparative effectiveness data to substantiate this view, remains a barrier [7].

Buprenorphine, which was approved for opioid use

Petro Petlovanyi, University of Zambia (UNZA), Zambia.

Gaise Kweku, Chainama Hills College Hospital, Zambia.

Evans Musonda, Chainama Hills College Hospital, Zambia. 
disorder in the USA in 2002, can be prescribed in officebased practices and is now usually given in a formulation with naloxone (BUP-NX) that provides some protection against misuse [7]. A study titled "Comparing overdose mortality associated with methadone and buprenorphine treatment" (2009) found that the risk of overdose death per thousand people in treatment was lower for buprenorphine than for methadone [9]. The short-term study concluded that, buprenorphine was associated with lower overdose risk than methadone. Frequent clinic visits are required during induction and throughout treatment [7].

A number of barriers contribute to low access to and utilization of MATs, including a paucity of trained prescribers and negative attitudes and misunderstandings about addiction medications held by the public, providers, and patients. For decades, a common concern has been that MATs merely replace one addiction with another. Many treatment-facility managers and staff favour an abstinence model, and provider scepticism may contribute to low adoption of MATs [10].

In this article, we describe our treatment of a patient with opioid use disorder with buprenorphine-naloxone. The informed consent for the patient was obtained for this report.

\section{CASE Study}

A 36-year-old male was admitted to our inpatient unit of Chainama Hills College Hospital in September, 2019. He stated that his substance abuse had started with the use of Pethidine at the age of 35 . He would inject pethidine once a day after work on a daily basis and would experience insomnia, diarrhoea, severe muscle pain and nervousness when he went a day without injecting Pethidine. In January, 2019 he enrolled for further studies in a different town where he tried to source for pethidine but could not afford it. He was at this point introduced to a gang where he began to access Heroin. He continued using Heroin despite being fully aware that it was causing negative consequences on his occupational, academic and marriage life. He however, did make unsuccessful attempts to cut down on use. Additionally, he would get upset if he was confronted on heroin use.

On the psychiatric assessment, opioid abstinence symptoms and severe craving for opioids were noted. He was diagnosed with opioid use disorder, severe, with physiological dependence based on the Diagnostic and Statistical Manual for Mental Disorders - Fifth Edition (DSM-V) [3]. Buprenorphine/Naloxone 4mg/1mg was commenced and dose was increased incrementally to $12 \mathrm{mg} / 3 \mathrm{mg}$ over 3 days. At this dose, the patient did not experience any cravings for heroin. A week later, the Buprenorphine/Naloxone taper was beginning at weekly decrements of $2 \mathrm{mg} / 0.5 \mathrm{mg}$ and currently, the patient is on a $2 \mathrm{mg} / 0.5 \mathrm{mg}$ daily dose. The patient has reported experiencing no cravings. The patient has now been on Buprenorphine/Naloxone for 15 weeks and urinalysis yields negative results for illicit drugs. He has been retained in the MAT program with weekly reviews. In addition, he has reported improved quality of life both in his marriage and academic life.

\section{Discussion: TREATMENT STRATEGIES}

The patient was prescribed an initial dose of $4 \mathrm{mg} / 1 \mathrm{mg}$ Buprenorphine/Naloxone which was increased to a dose of $12 \mathrm{mg} / 3 \mathrm{mg}$ over 3 days to prevent cravings. The starting dose for the induction phase was based on the FDA approved dose range of 4-24 $\mathrm{mg}$ of buprenorphine and the $4 \mathrm{mg} / 1 \mathrm{mg}$ increment daily over three days was based on recommendations that the dose can be increased in 2-4 mg increments over the next 2-3 days to a dose that eliminates any further craving or withdrawal symptoms [11]. After one week of treatment, the patient had no withdrawal symptoms and no cravings for heroin. The patient then opted for a buprenorphine/ naloxone taper at weekly decrements of $2 \mathrm{mg} / 0.5 \mathrm{mg}$. This was based on guidance from literature that recommends subsequent adjustment of buprenorphine/naloxone dose in increments or decrements of $2 \mathrm{mg} / 0.5 \mathrm{mg}$ or $4 \mathrm{mg} / 1 \mathrm{mg}$ to level that holds patient in treatment and suppresses opioid withdrawal signs and symptoms for maintenance phase of treatment [12].

The buprenorphine/naloxone taper was done over a period of 98 days (14 weeks) based on the evidence that gradual reduction in buprenorphine dose after detoxification is likely to produce superior treatment outcomes than more rapid buprenorphine tapers [13]. This evidence comes from the study where two detoxification schedules of buprenorphine were compared: buprenorphine gradual (36 days; $\mathrm{N}=3$ ) or buprenorphine rapid (12 days; $\mathrm{N}=5$ ) and found that a 36-day, gradual buprenorphine detoxification was more successful treatment than a 12-day, rapid buprenorphine detoxification [13]. Individuals receiving the gradual detoxification achieved longer periods of continuous opioid abstinence, produced a higher percentage of opioid-negative urines, reported less withdrawal and had better treatment retention than individuals receiving the rapid detoxification [13].

\section{Discussion: Clinical USE of BUPRENORPHINE/NALOXONE}

When using buprenorphine/naloxone, it is important to understand the drugs pharmacology. As a partial opioid agonist, it has a very high affinity for the mu opioid receptor, and it will displace most other opioid agonists. Because it does not fully activate the receptor, any individual physically dependent on a full opioid agonist will experience this displacement as the acute onset or severe worsening of opioid withdrawal symptoms, a syndrome called "precipitated withdrawal." This severe but brief syndrome is comparable to the effects of treatment with an opioid antagonist [11]. During induction, the most important task is to introduce the medication while not causing precipitated withdrawal. It is advised that this problem be avoided by waiting until the patient is opioid free or already in mild to moderate opioid withdrawal before administering the first dose of buprenorphine [11]. This is best accomplished by advising the patient to avoid any opioids for 12-24 hours for short acting opioids and at least 24 hours for long acting opioids prior to induction and by documentation of the presence of withdrawal signs using a standard scale such as the Clinical Opiate Withdrawal Scale (COWs) for Measuring Symptoms. The COWs for Measuring Symptoms scores patients on 
observable measures including pulse, sweating, restlessness, pupil size, tremor, yawning, runny nose/tearing, and goose flesh and more subjective measures such as bone or joint aches and abdominal cramps, permitting a reliable measure of the severity of withdrawal [11]. Maintenance therapy involves a sublingual or buccal combination of buprenorphine and the short-acting opioid antagonist naloxone, usually in a 4-to-1 ratio across the two drugs [14]. This ensures a diminished risk of diversion and abuse for the buprenorphine-naloxone. Due to the low doses of naloxone administered and the low proportion of this drug that is absorbed orally, this opioid antagonist does not precipitate withdrawal unless it is injected intravenously, in which case the withdrawal symptoms can be sudden and severe [15].

\section{Discussion: Treatment OUtCOMES OF OPIOID USE Disorder}

The main outcomes reported randomised controlled trials include: Proportion of negative urine opioid screenings, Selfreported substance use (opiate, amphetamines, cannabis, benzodiazepines, and alcohol), Retention into treatment, Adverse effects, Opiate Withdrawal (using both subjective and objective scales), cravings and sedation (using visual analogue scales) [16]. In the case at hand the outcomes assessed were craving and sedation especially in the induction phase while other measures like negative urine opioid screening, retention into treatment and duration of stay in treatment were assessed in the maintenance phase of the treatment. Additional monitoring was for the craving and withdraw symptoms during the buprenorphine taper. It should be noted that at the time of writing this article, the patient had spent 98 days in treatment and during this period he had not used heroin nor any other illicit drugs. It must be emphasised however, that the long-term outcome of withdrawal treatment is rarely good as it is associated with a relapse rate of 80-93\% within 1 year following a brief inpatient taper [11]. The best results have been seen with very prolonged outpatient tapers or 1-2 week inpatient tapers followed by long-term residential treatment [11]. In this regard, patients must be warned about these risks if withdrawal treatment is not followed by long-term residential care or treatment with long term outpatient taper.

\section{CONCLUSIONS}

Opioid use disorder is often a chronic, relapsing condition associated with increased morbidity and death. It affects the health, economic, social aspects of a human being, the community, nation and the world at large. However, with appropriate treatment and follow-up, individuals can reach sustained long-term remission. Effective long-term treatment of opioid dependence often requires multiple treatment episodes and a combination of different responses. Harm reduction interventions, mental health services and other services that address comorbid mental and physical health problems are also important.

\section{REFERENCES}

[1] United Nations Office on Drugs and Crime. (2019). WORLD DRUG REPORT 2019 (SET OF 5 BOOKLETS). S.1.: UNITED NATIONS.

[2] Fischer, B. (2005). Illicit Opioid Use in Canada: Comparing Social, Health, and Drug Use Characteristics of Untreated Users in Five Cities (OPICAN Study). Journal of Urban Health: Bulletin of the New York Academy of Medicine, 82(2), 250-266. https://doi.org/10.1093/jurban/jti049

[3] Diagnostic and statistical manual of mental disorders: DSM-5TM, 5th ed. (2013). https://doi.org/10.1176/appi.books.9780890425596

[4] Volkow, N. D., Frieden, T. R., Hyde, P. S., \& Cha, S. S. (2014). Medication-Assisted Therapies-Tackling the Opioid-Overdose Epidemic. New England Journal of Medicine, 370(22), 2063-2066. https://doi.org/10.1056/NEJMp1402780

[5] Stotts, A. L., Dodrill, C. L., \& Kosten, T. R. (2009). Opioid dependence treatment: Options in pharmacotherapy. Expert Opinion on Pharmacotherapy, $10(11), \quad 1727-1740$. https://doi.org/10.1517/14656560903037168

[6] Lev, R., Petro, S., Lee, A., Lee, O., Lucas, J., Castillo, E. M., and Vilke, G. M. (2015). Methadone related deaths compared to all prescription related deaths. Forensic Science International, 257, 347-352. https://doi.org/10.1016/j.forsciint.2015.09.021

[7] Volkow, N. D. (2018). Medications for opioid use disorder: Bridging the gap in care. The Lancet, 391(10118), 285-287. https://doi.org/10.1016/S0140-6736(17)32893-3

[8] Maradiaga, J. A., Nahvi, S., Cunningham, C. O., Sanchez, J., \& Fox, A. D. (2016). 'I Kicked the Hard Way. I Got Incarcerated.' Withdrawal from Methadone During Incarceration and Subsequent Aversion to Medication Assisted Treatments. Journal of Substance Abuse Treatment, 62, 49-54. https://doi.org/10.1016/j.jsat.2015.11.004

[9] Bell, J. R., Butler, B., Lawrance, A., Batey, R., \& Salmelainen, P. (2009). Comparing overdose mortality associated with methadone and buprenorphine treatment. Drug and Alcohol Dependence, 104(1-2), 73-77.

[10] Knudsen, H. K., Abraham, A. J., \& Roman, P. M. (2011). Adoption and Implementation of Medications in Addiction Treatment Programs. Journal of Addiction Medicine, 5(1), 21-27. https://doi.org/10.1097/ADM.0b013e3181d41ddb

[11] Kelly, J. F., \& Wakeman, S. E. (2019). Treating opioid addiction. Retrieved from https://search.ebscohost.com/login.aspx?direct=true\&scope=site \&db= nlebk\&db=nlabk\&AN=2200017

[12] Suboxone, Zubsolv (buprenorphine/naloxone) dosing, indications, interactions, adverse effects, and more. (n.d.). Retrieved 25 November 2019, from https://reference.medscape.com/drug/suboxone-zubsolvbuprenorphine-naloxone-343334

[13] Amass, L., Bickel, W. K., Higgins, S. T., \& Hughes, J. R. (1995). A Preliminary Investigation of Outcome Following Gradual or Rapid Buprenorphine Detoxification. Journal of Addictive Diseases, 13(3), 33-45. https://doi.org/10.1300/J069v13n03_04

[14] Soyka, M. (2015). New developments in the management of opioid dependence: Focus on sublingual buprenorphine \& naloxone. https://doi.org/10.2147/SAR.S45585

[15] Schuckit, M. A. (2016). Treatment of Opioid-Use Disorders.

[16] Buprenorphine Formulations for the Treatment of Opioid Use Disorders: A Review of Comparative Clinical Effectiveness, Cost Effectiveness and Guidelines. (n.d.). https://www-ncbi-nlm-nihgov.ezp01.library.qut.edu.au/books/NBK525042/pdf/Bookshelf_NBK 525042.pdf 